\title{
Organobismuth Redox Manifolds: Versatile Tools for Synthesis
}

\author{
Katie Ruffell and Liam T. Ball* \\ School of Chemistry, University of Nottingham, University Park, Nottingham NG7 2RD, U.K. \\ liam.ball@nottingham.ac.uk \\ orcid: 0000-0003-3849-9006
}

\begin{abstract}
Bismuth's ability to manoeuvre between oxidation states supports several distinct reaction manifolds. Recent advances in the design, synthesis and application of organobismuth reagents and catalysts illustrate the potential of these redox manifolds as complementary tools to conventional transition metal-based synthesis strategies.
\end{abstract}

The ease with which transition metals can cycle between different oxidation states, and the extent to which this reactivity can be tuned by judicious ligand design, is central to many homogeneous catalytic processes. Extension of the same principles to metals from beyond the d-block not only allows known reactivity manifolds to be replicated with cheaper or less toxic elements, but also has the potential to unlock new and exciting opportunities for synthesis. ${ }^{1}$

With four easily accessible oxidation states $(+1,+2,+3$ and +5$)$, bismuth presents a diversity of 1- and 2-electron redox couples ripe for exploitation in synthesis. ${ }^{2}$ Following pioneering work by Barton in the 1980 s, $^{3}$ a recent resurgence of interest in organobismuth chemistry serves to highlight the current utility of this non-toxic, cheap metal, and the future promise that it holds.

In 2017, Coles et al. reported a catalytic $\mathrm{Bi}(\mathrm{II}) / \mathrm{Bi}(\mathrm{III})$ manifold for the oxidative coupling of TEMPO and phenylsilane (Figure 1a). ${ }^{4}$ Informed by stoichiometric studies and isolation of putative intermediates, the mechanism is proposed to proceed via reaction of a bis(amido)disiloxane Bi(II) species (1a or $\mathbf{1 b}$ ) with TEMPO to give a $\mathrm{Bi}(\mathrm{III})$ TEMPOxide, followed by product-forming $\mathrm{Bi}-\mathrm{O} / \mathrm{Si}-\mathrm{H}$ metathesis with phenyl silane. The resulting $\mathrm{Bi}(\mathrm{III})$ hydride readily extrudes $\mathrm{H}_{2}$ to regenerate the $\mathrm{Bi}(\mathrm{II})$ species and complete the catalytic cycle. This work represents the first example of dehydrosilylation reactivity by a heavy main group element, and is the first well-substantiated example of homogeneous bismuth redox catalysis. 
a) Coles - Bi-catalysed oxidative coupling of TEMPO with phenyl silane

$$
\mathrm{Ph}^{-} \mathrm{SiH}_{3} \stackrel{\begin{array}{c}
\text { 1a or } \mathbf{1 b}(10 \mathrm{~mol} \%) \\
\text { TEMPO }(1.0 \text { eq. })
\end{array}}{\stackrel{\mathrm{C}_{6} \mathrm{D}_{6}, 70^{\circ} \mathrm{C}, 15 \mathrm{~d}}{\longrightarrow}}
$$

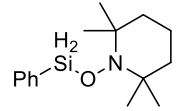

Bi platform

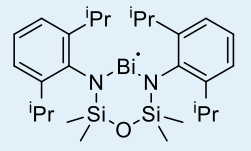

$1 \mathrm{a}$

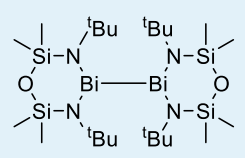

$[1 \mathrm{~b}]_{2}$

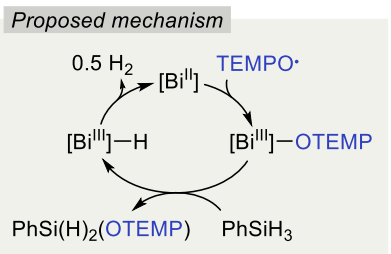

$\mathrm{PhSi}(\mathrm{H})_{2}$ (OTEMP) $\quad \mathrm{PhSiH}_{3}$ c) Ball - Bi-mediated selective $\mathrm{C}$-H arylation of phenols and naphthols

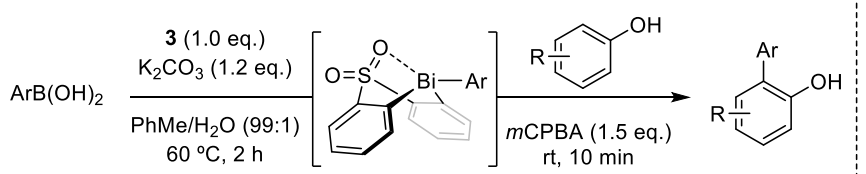
$60^{\circ} \mathrm{C}, 2 \mathrm{~h}$
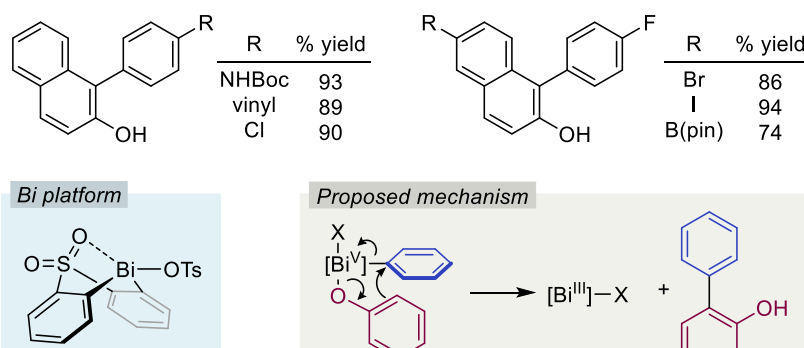

3

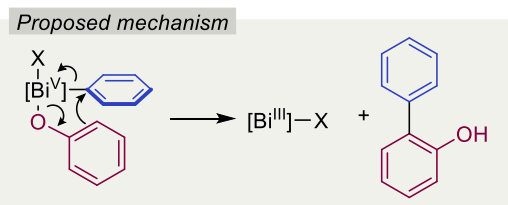

b) Cornella - Bi-catalysed transfer-hydrogenation with ammonia-borane
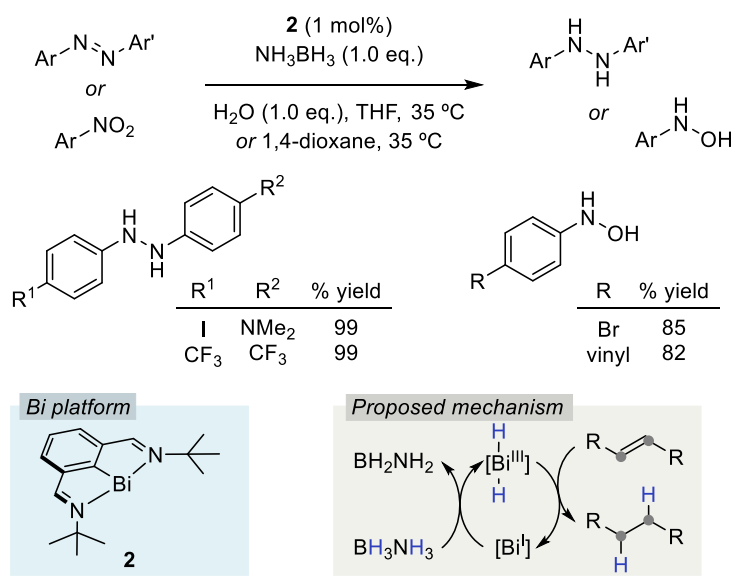

d) Cornella - Bi-catalysed fluorination of arylboronic esters
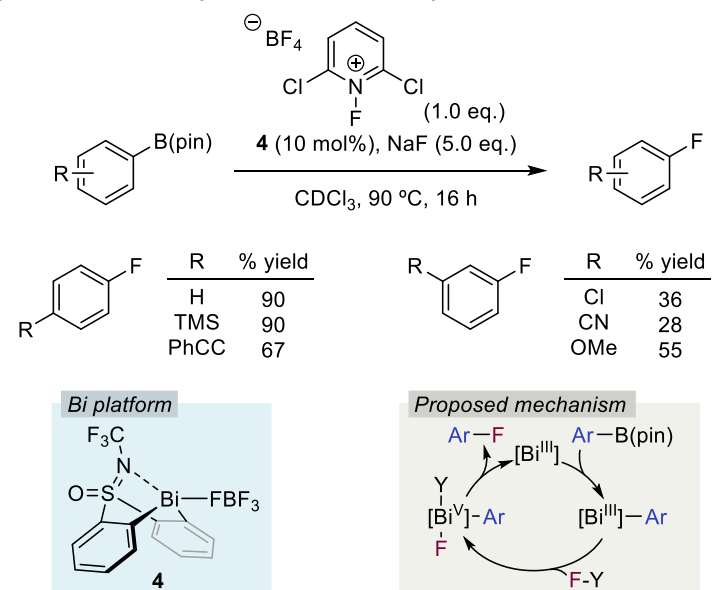

Figure 1. Diverse organobismuth redox manifolds and ligand architectures for synthesis.

Cornella et al. subsequently reported a $\mathrm{Bi}(\mathrm{I}) / \mathrm{Bi}(\mathrm{III})$ redox manifold for the catalytic transfer-hydrogenation of azoand nitroarenes with ammonia-borane (Figure 1b). ${ }^{5}$ Similar to Cole's work, ${ }^{4}$ this catalytic process is driven by the high reactivity of $\mathrm{Bi}(\mathrm{III})$ hydride species. Here, it is proposed that oxidation of $N, C, N$-chelated bismuthinidene 2 by ammonia-borane forms a $\mathrm{Bi}(\mathrm{III})$ dihydride intermediate which mediates the reduction of azoarenes and nitroarenes. The protocol exhibits broad scope and tolerates functionality that is incompatible with transition metal catalysed methods. Furthermore, in contrast to the majority of transition metal catalysed hydrogenations, the reduction of nitroarenes occurs with high selectivity towards the $\mathrm{N}$-arylhydroxylamine products. Thus, this work provides a clear demonstration of the complementarity that exists between many conventional synthesis methods and those mediated by bismuth.

Recently, our group reported a highly modular protocol for the bismuth-mediated, ortho-selective C-H arylation of phenols and naphthols using boronic acids (Figure 1C). ${ }^{6}$ Arylation is achieved via a telescoped process of (1) Bto-Bi transmetalation from an arylboronic acid to the bench-stable Bi(III) precursor 3, (2) oxidation of the resulting aryl bismacycle with $m C P B A$, and $(3)$ subsequent generation of a $\mathrm{Bi}(\mathrm{V})$ phenoxide that undergoes product-forming reductive ligand coupling. The success of this $\mathrm{Bi}(\mathrm{III}) / \mathrm{Bi}(\mathrm{V})$ manifold depends on use of a bidentate diphenylsulfone scaffold that prevents ligand scrambling, ${ }^{7}$ enables selective transfer of the exocyclic aryl group, and improves atom efficiency by facilitating recovery and recycling of the bismacycle. The methodology provides facile access to 2-hydroxybiaryls without pre-functionalisation of the phenol, exhibits excellent functional group tolerance, and is orthogonal to conventional transition metal based cross coupling strategies.

The $\mathrm{Bi}(\mathrm{III}) / \mathrm{Bi}(\mathrm{V})$ manifold has also been exploited by Cornella et al. for the fluorination of arylboronic esters (Figure 1d). ${ }^{8}$ The catalytic variant of this powerful methodology proceeds via B-to-Bi transmetalation from an arylboronic ester to bismacycle 4, prior to oxidative fluorination of the resulting arylbismuth(III) species. Subsequent reductive ligand coupling closes the cycle, and is enabled by a bespoke, electron-deficient bismacycle 
scaffold that drives the challenging C-F bond formation. This protocol represents a convenient tool for the formation of important C-F bonds, a transformation which remains non-trivial for transition metal catalysts, and once again provides orthogonal functional group compatibility relative to conventional systems.

The recent advances in synthetic applications have been paralleled by advances in the design, preparation and understanding of ligands for bismuth. The opportunity to utilise ligands to tune the metal centre was largely overlooked in early work, which focussed primarily on the use of homoleptic triarylbismuthanes. However, it is now clear that ligand design is crucial for ensuring access to stable, practical bismuth reagents and for modulating their redox windows. As an illustration of this latter point, Chitnis et al. recently reported a redox-confused bismuth (I/III) triamide formed by complexation of a $\mathrm{Bi}(\mathrm{III})$ salt with a redox non-innocent tridentate ligand (Figure 2a). ${ }^{9}$ Calculations suggest that the electronic structure is best represented by $\mathrm{Bi}(\mathrm{I})$ resonance form $\mathbf{5 B}$; however, non-negligible contributions from resonance form $5 \mathrm{~A}$ means that the species also possesses $\mathrm{Bi}(\mathrm{III})$ character, resulting in ambiphilic reactivity and redox-confused $\mathrm{Bi}(\mathrm{I} / \mathrm{III})$ behaviour.
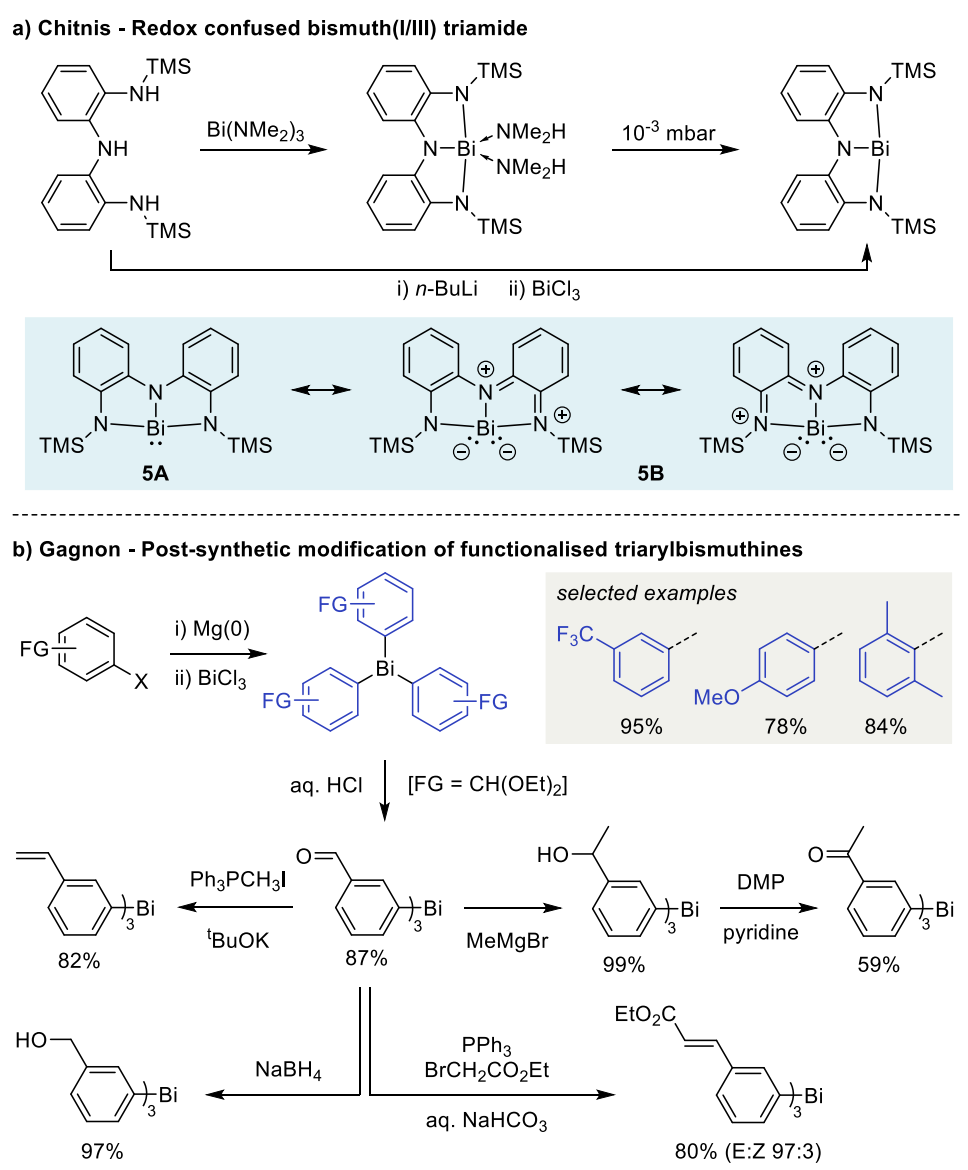

Figure 2. Increased diversity and understanding of organobismuth architectures will underpin future synthetic applications.

For methodologies that rely on arylation (e.g., Figure $\mathbf{1 c}$ and $\mathbf{1 d}$ ), it is essential that a third, unique aryl group can be installed at bismuth. Recent advances in B-to-Bi transmetallation ${ }^{6,8}$ provide convenient protocols for the installation of aryl and heteroaryl groups onto a bismuth scaffold using arylboron reagents. Alternatively, Gagnon et al. reported a complementary strategy in the context of post-synthetic modification of simple homoleptic triarylbismuthanes. Highly functionalised triarylbismuthanes bearing primary, secondary and tertiary alcohols, aldehydes, $\alpha, \beta$-unsaturated esters, vinyl substituents and methyl ketones can be prepared via functional group manipulation (Figure $\mathbf{2 b}$ ). ${ }^{10}$ Though not explicitly demonstrated, this methodology also enables bismuth redox windows to be tuned by judicious post-synthetic functionalisation of ligand scaffolds, an area of significant but under-explored potential.

As demonstrated by the diversity of transformations presented in recent publications, the ability of bismuth to occupy different redox manifolds represents a rich and varied toolbox for synthesis. These new methodologies 
illustrate the dual ability of bismuth to mimic and to complement reactivity commonly associated with transition metal-based systems. Detailed mechanistic studies performed by $\mathrm{Hyvl}^{7}$, Cornella ${ }^{5,8}$ and our group ${ }^{6}$ provide important fundamental insight into bismuth redox chemistry, and will likely underpin the next generation of rationally-designed systems. Recent advances in the synthesis of bismuth reagents allow for more convenient access, use and modulation of the metal's properties. It is anticipated that, by building on these foundations, organobismuth redox chemistry will continue to be the source of exciting new reactivity manifolds and synthesis tools for future applications.

\section{References}

1 P. P. Power, Nature, 2010, 463, 171-177.

2 A. Gagnon, J. Dansereau and A. Le Roch, Synth., 2017, 49, 1707-1745.

3 D. H. R. Barton, D. J. Lester, W. B. Motherwell and M. T. B. Papoula, J. Chem. Soc., Chem. Commun., 1980, 246-247.

4 R. J. Schwamm, M. Lein, M. P. Coles and C. M. Fitchett, Chem. Commun., 2018, 54, 916-919.

5 F. Wang, O. Planas and J. Cornella, J. Am. Chem. Soc., 2019, 141, 4235-4240.

6 M. Jurrat, L. Maggi, W. Lewis and L. T. Ball, Nat. Chem., 2020, 12, 260-269.

7 T. Louis-Goff, A. L. Rheingold and J. Hyvl, Organometallics, 2020, 39, 778-782.

8 O. Planas, F. Wang, M. Leutzsch and J. Cornella, Science (80-. )., 2020, 367, 313-317.

9 M. B. Kindervater, K. M. Marczenko, U. Werner-Zwanziger and S. S. Chitnis, Angew. Chemie Int. Ed., 2019, 58, 7850-7855.

10 M. Hébert, P. Petiot, E. Benoit, J. Dansereau, T. Ahmad, A. Le Roch, X. Ottenwaelder and A. Gagnon, J. Org. Chem., 2016, 81, 5401-5416. 